\title{
The Cornell Caltech Atacama Telescope status and technical progress
}

Thomas A. Sebring, Simon Radford, Riccardo Giovanelli, Jason Glenn, David Woody

Thomas A. Sebring, Simon Radford, Riccardo Giovanelli, Jason Glenn, David Woody, "The Cornell Caltech Atacama Telescope status and technical progress," Proc. SPIE 7012, Ground-based and Airborne Telescopes II, 70121H (10 July 2008); doi: 10.1117/12.787586

Event: SPIE Astronomical Telescopes + Instrumentation, 2008, Marseille, France 


\title{
The Cornell Caltech Atacama Telescope Status and Technical Progress
}

\author{
Thomas A. Sebring*a , Simon Radford ${ }^{\mathrm{b}}$, Riccardo Giovanelli ${ }^{\mathrm{a},}$ Jason Glenn $^{\mathrm{c}}$, David Woody ${ }^{\mathrm{d}}$ \\ ${ }^{\mathrm{a}}$ Center for Radiophysics and Space Research, Cornell University, Ithaca, NY USA 14853; \\ ${ }^{\mathrm{b}}$ Submillimeter Astronomy, California Institute of Technology, Pasadena, CA USA 91125; \\ ${ }^{\mathrm{c}}$ Department of Astrophysical and Planetary Science, University of Colorado, Boulder, CO \\ 80309 \\ ${ }^{\mathrm{d}}$ OVRO, California Institute of Technology, Pasadena, CA USA 91125
}

\begin{abstract}
Five partners have currently joined a Consortium to develop the Cornell Caltech Atacama Telescope (CCAT.) Included are Cornell University, the California Institute of Technology (Caltech), the University of Colorado at Boulder, the United Kingdom as represented by the Astronomy Technology Centre (ATC), and Canada as represented by the Universities of British Columbia and Waterloo. This consortium has continued work toward the design of the telescope and instrumentation, pursued fund raising, and further developed the science case for CCAT. An Engineering Design Phase is being planned for 2009-2011 with construction planned to begin shortly thereafter. CCAT continues as a wide field (20 arc min) FOV telescope operating from a shortest wavelength of $200 \mu$. Testing has continued near the summit of Cerro Chajnantor in the Atacama Region of Chile above 5600 meters altitude and data indicates significantly lower water vapor in the seeing column than measured at the ALMA site on the plateau below. Work over the past two years has included research on manufacturing methods for optical segments, extensive study of mirror alignment sensing and control techniques, additional concepts for major structures, and further development of instrumentation.
\end{abstract}

\section{INTRODUCTION}

Five partners including Cornell, Caltech, U. Colorado, the UK, and Canada have formed a consortium to pursue construction of a 25 meter telescope operating down tot $200 \mu$ wavelength at an altitude above 5600 meters in the Atacama Desert of Chile. CCAT will be a seminal tool for astronomical science as it will employ the recent emergence of wide area superconducting multi-pixel bolometer arrays such as Transition Edge Sensors (TES) and Microwave Kinetic Inductance Detectors to enable rapid surveys, detecting millions of as yet undiscovered submillimeter galaxies and providing new information on early history of the cosmos. A Feasibility Concept Design Study was completed in 2006 and since then several new partners have joined the Consortium with others signifying their intent to join. Work over the next two years will focus on refining the design of CCAT leading to the inception of the construction phase.

\section{BACKGROUND}

The concept for CCAT presented in the Feasibility Concept Design Study provided a top-level approach to the design and construction of the telescope and instrumentation. The study was funded by Cornell and Caltech, took approximately 18 months to complete, and was favorably reviewed by an independent blue-ribbon panel of astronomers. Subsequently work focused on building the partnership and to date the Consortium is adequately subscribed to contemplate construction of CCAT. At the same time this process has been pursued additional technical work has proceeded on a number of fronts. Based on the input from the review panel and the assessment of the CCAT Partners, the key technological and programmatic risks have been identified and work has progressed in these areas using both contractor and in-house resources. Development of the science case and, in particular, science instrumentation has progressed as well. The specific technical accomplishments realized over the past two years are discussed in some detail in the following sections.

*sebring@astro.cornell.edu; phone 1607255 1278; fax 16072559002

Ground-based and Airborne Telescopes II, edited by Larry M. Stepp, Roberto Gilmozzi, Proc. of SPIE Vol. 7012, 70121H, (2008) · 0277-786X/08/\$18 - doi: 10.1117/12.787586 


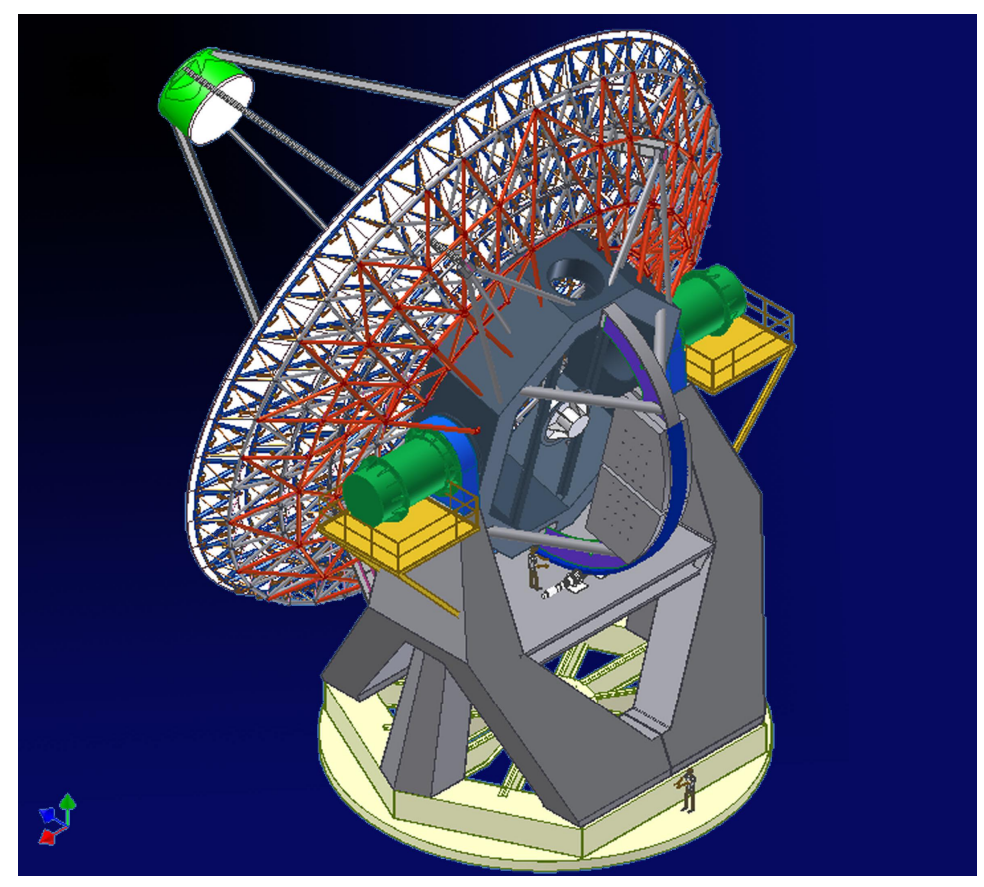

Fig. 1. CCAT Telescope Concept by Vertex RSI Texas.

CCAT is a Ritchey Chretien design offering two Nasmyth and two bent Cassegrain instrument locations. The telescope requirements are summarized.

Table 1. Telescope Requirements. [1]

\begin{tabular}{|c|c|c|c|}
\hline & Requirement & Goal & Remark \\
\hline Wavelength & $350-1400$ & $200-2500$ & $\mu \mathrm{m}$ \\
\hline Aperture & $25 \mathrm{~m}$ & & \\
\hline Field of view & $10^{\prime}$ & $20^{\prime}$ & \\
\hline Half WFE & $<12.5 \mu \mathrm{m}$ & $<9.5 \mu \mathrm{m}$ & $\mathrm{Rms}$ \\
\hline Site Condns. & $<1.0 \mathrm{~mm}$ & $<0.7 \mathrm{~mm}$ & Median pwv \\
\hline Polarization & $0.2 \%$ & $0.05 \%$ & After cal. \\
\hline Emissivity & $\begin{array}{l}<10 \% @>300 \mu \mathrm{m} \\
<20 \% @ 200 \mu \mathrm{m}\end{array}$ & $<5 \% @>800 \mu \mathrm{m}$ & \\
\hline Pntg, blind & $2 "$ & $0.5^{\prime \prime}$ & Rms \\
\hline Pntg, offset & $0.3^{\prime \prime}$ & $0.2^{\prime \prime}$ & Within $1^{\circ}$ \\
\hline Pntg, repeat & $0.3^{\prime \prime}$ & $0.2^{\prime \prime}$ & Rms, 1 hour \\
\hline Scanning rate & $0.2^{\circ} \mathrm{s}^{-1-}$ & $1^{\mathrm{o}} \mathrm{s}^{-\mathrm{T}^{-}}$ & Slow/fast \\
\hline Scan. accel. & $0.4^{\mathrm{o}} \mathrm{s}^{-2}$ & $2^{\circ} \mathrm{s}^{-2}$ & Short/long $\lambda$ \\
\hline Pointing knowledge & $0.2^{\prime \prime}$ & $0.1^{\prime \prime}$ & Rms \\
\hline M2 nutation & $\pm 2.5^{\prime} @ 1 \mathrm{~Hz}$ & & Azimuth onl \\
\hline
\end{tabular}

The telescope site is a plateau on the eastern side of Cerrro Chajnantor approximately $50 \mathrm{M}$ altitude below the summit. Access to the site is provided by a road constructed by the Tokyo Astronomical Observatory. The telescope uses a segmented primary mirror with segment positions actively controlled similarly to the optical segmented telescopes such as Keck and HET. 


\section{SCIENCE}

Plans for science with CCAT continue to evolve. The basic mission addresses deep, large area, multi-color submillimeter surveys which also serve to find objects of interest that can profitably be addressed with the Atacama Large Millimeter Array (ALMA). "CCAT observations will address fundamental themes in contemporary astronomy, notably the formation and evolution of galaxies, the nature of the dark matter and dark energy that comprise most of the content of the universe, the formation of stars and planets, the conditions in circumstellar disks, and the conditions during the early history of the Solar system." ${ }^{[2]}$

With its large focal plane array cameras, CCAT will generate vast catalogs of galaxies, ranging from the local universe to the earliest epoch of galaxy formation at high redshift. Additionally, early indications from the flux-limited $1.1 \mathrm{~mm}$ Caltech Submillimeter Observatory Bolocam Galactic Plane Survey indicate that a CCAT Galactic plane survey would yield tens of thousands of sites of star formation (detected by their thermal dust emission). Indeed, when CCAT is commissioned formidable submillimeter source catalogs already will have been generated by the Herschel Space Observatory and by SCUBA-2. Spectroscopic capability for CCAT will be essential to probe the gas-phase astrophysics of these sources. ALMA will provide follow-up of CCAT sources; however, ALMA will be heavily subscribed and in two important regimes CCAT could provide more rapid follow up. First, with a heterodyne array receiver of 50 or more pixels at a frequency of $850 \mathrm{GHz}$ (or above), CCAT will be able to map molecular clouds (and nearby galaxies), without loss of zero-spacing information. Second, multi-object spectroscopic capability for point sources in the $350 \mu \mathrm{m}$ atmospheric window will allow for simultaneous redshift measurements of a dozen or more submillimeter galaxies without the need for optical or radio counterparts (Figure 1).

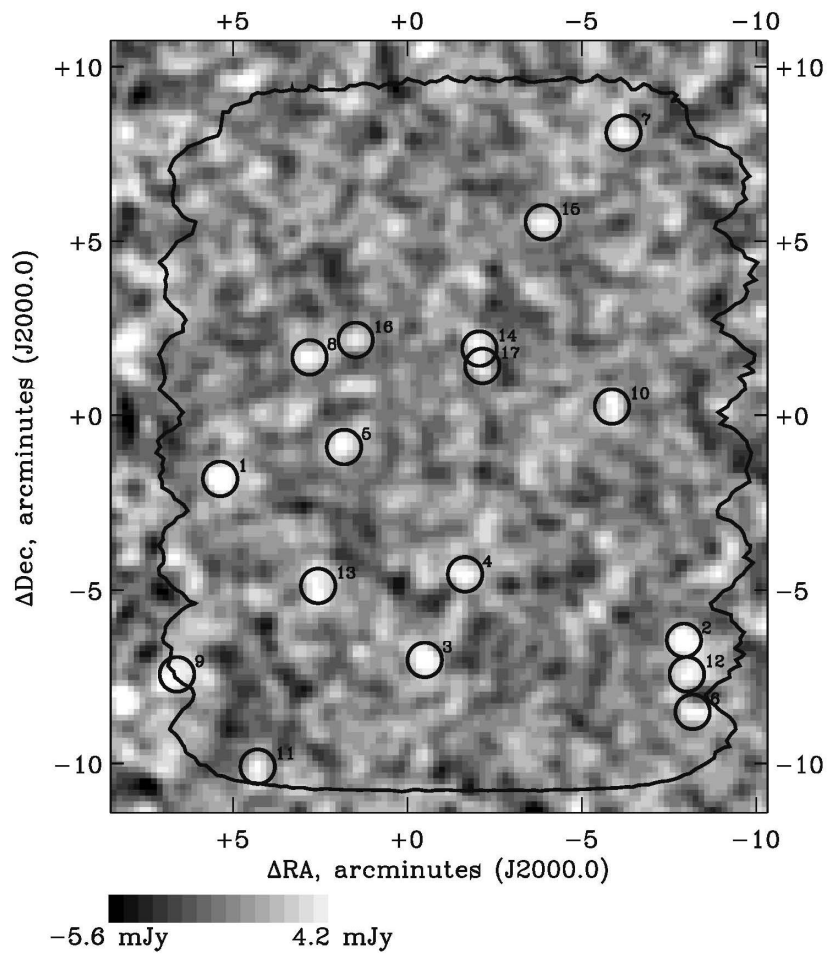

Figure 2. A Bolocam $1.1 \mathrm{~mm}$ continuum map of the Lockman Hole East region. The region interior to the irregular line well matched to the 20 arcminute planned field of view of CCAT - is the low/uniform-noise region of the map. Circles indicate galaxy candidates and could represent positions of fiber inputs in the CCAT focal plane for a multi-object spectrograph (closely spaced galaxies would require subsequent observations to avoid fiber overlap). The Bolocam beam is 30 arcseconds FWHM; thus, the galaxies are unresolved in this image. ${ }^{[3]}$

The necessary technologies required for submillimeter multi-object spectroscopy are in-hand or under development. ZEUS $^{[4]}$ achieves a high spectral resolution by combining an echelle grating with pop-up bolometers. The marriage of bolometers and a waveguide-coupled diffraction grating has been achieved in Z-Spec ${ }^{[5]}$, which is being used for line 
surveys of ultra-luminous infrared galaxies and redshift measurements of distant galaxies. By virtue of being waveguide coupled, and therefore very thin in the cross dispersion direction, Z-Spec's grating configuration would lend itself naturally to multi-object spectroscopy by stacking gratings. Each grating could be "fed" by a movable waveguide with its entrance aperture at CCAT's focal plane and terminated at the input to a waveguide-coupled grating. One promising technology for flexible waveguides involves metallizing the interiors of hollow tubing, such as polycarbonate (Figure 2). Several thousand detectors will be required to enable multi-object capability with spectral resolution of $\mathrm{R} \approx 1,000$. It is likely that both Transition-Edge Sensors $\left(\mathrm{TESs}^{[6]}\right.$ ) and Microwave Kinetic Inductance Detectors (MKIDs ${ }^{[7]}$ ) will be sufficiently mature technologies.

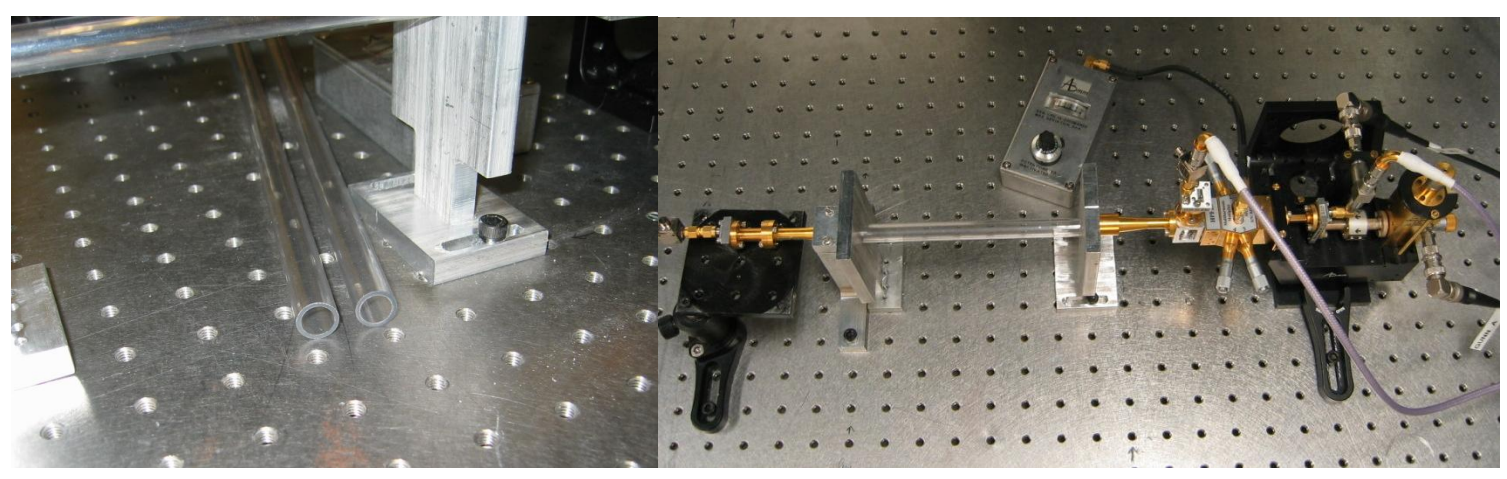

Fig. 3. Photographs of a setup for low frequency, scale model testing of hollow flexible waveguides. The left panel shows a close-up of two guides and the right panel shows a waveguide in the test configuration. ${ }^{[8]}$

\section{OPTICAL DESIGN}

One of the design trade space issues considered was whether the telescope could be made more compact (lower f/ratio), leaning more towards radio rather than optical telescope design. In terms of the CCAT science objectives and instrumentation requirements, any primary focal ratio, $f$, between 0.4 and 0.6 (the original CCAT f/ratio) is equivalent. With a smaller focal ratio, the secondary mirror and dome would be smaller. For example, the secondary mirror diameter is $2.62 \mathrm{~m}$ when $f=0.4$ rather than $3.2 \mathrm{~m}$ when $f=0.6$. The positioning tolerances of the primary mirror panels and secondary mirror are tighter for a smaller focal ratio. Analysis of the sensitivity/tolerance changes over this range (many of which scale as $f^{2}$ ) is given.

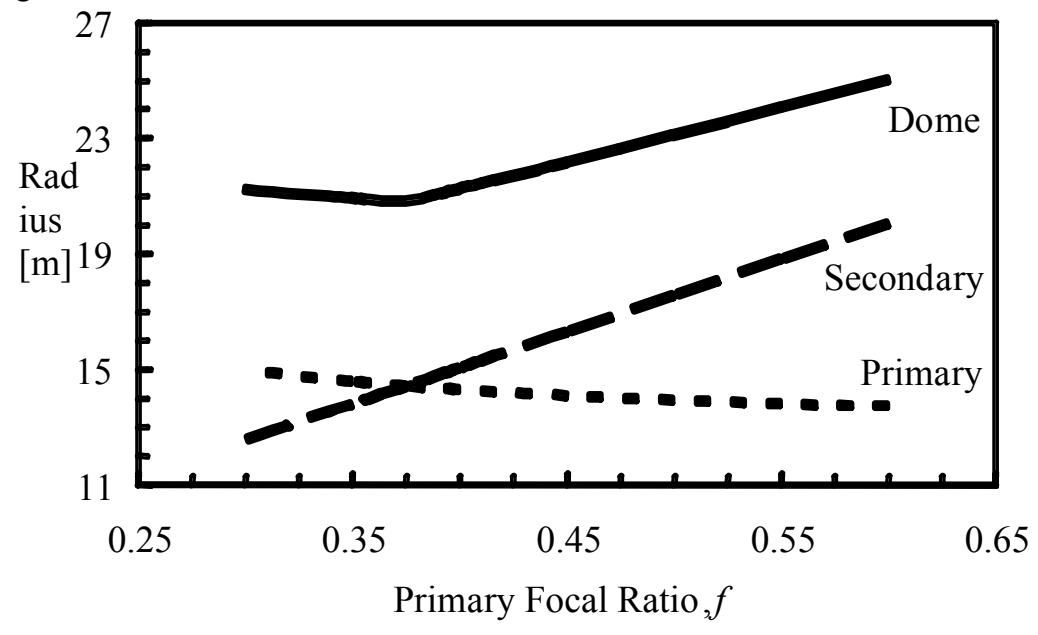

Fig 4. Radii of the dome and of the volumes swept by the primary mirror and the secondary mirror support for different primary focal ratios (Simon Radford). 
Historically, the cost of the enclosure dome depends strongly on size, perhaps as diameter ${ }^{2.6}$. Hence a $42 \mathrm{~m}$ diameter dome might be as much as $35 \%$ less expensive as a $50 \mathrm{~m}$ dome.

A faster primary mirror has other advantages. Because the telescope is more compact, the secondary mirror support legs will be shorter. These are representative of the round trip path any metrology system must measure. If the metrology system has a fixed relative accuracy, then the absolute accuracy will be finer on a shorter path. Reducing the focal ratio from 0.6 to 0.4 reduces the representative metrology path by about $20 \%$.

The CCAT Concept Feasibility Study report contains an extensive analysis of the optical performance of the baseline telescope design $(f=0.6)$. Further optical analysis shows a faster $(f=0.4)$ alternative:

- is more compact;

- requires a smaller secondary mirror (2.6 m vs. $3.2 \mathrm{~m})$, resulting in less blockage ( $1.0 \%$ vs. $1.5 \%)$;

- has a more curved focal surface (1.1 m radius vs. $1.9 \mathrm{~m}$, Figure 2$)$;

- has a slightly lower, but still very good, Strehl ratio ( $\geq 95 \%$ vs. $\geq 97 \%$ ) at $200 \mu \mathrm{m}$ over the entire $20^{\prime}$ field of view (FoV);

- has a smaller FoV with a flat focal surface (5.7' vs. 8.0' at $200 \mu \mathrm{m}$ for Strehl ratio $\geq 95 \%$, Figure 3);

- requires a tighter secondary mirror focus tolerance for high quality images (45 $\mu \mathrm{m}$ vs. $80 \mu \mathrm{m}$ for Strehl ratio $\geq$ $95 \%$, Figure 4);

- requires tighter secondary mirror lateral displacement tolerances for pointing (14 $\mu \mathrm{m}$ vs. $18 \mu \mathrm{m}$, Figure 5); and

- permits only smaller secondary mirror nutation angles ( \pm 4 beams vs. \pm 8 beams; Figure 6 ).

- Is more sensitive to misalignment, though still within acceptable limits.

It was resolved to accept the faster f/ratio and the new prescription is the current CCAT baseline.

\section{FACILITY AND SITE}

Cerro Chajnantor is the intended site for CCAT. The CCAT site is approximately 50m altitude below the summit, where the Tokyo Astronomical Observatory (TAO) has announced intentions to place a $6.5 \mathrm{~m}$ infrared telescope. Figure 4 shows the topography of the CCAT site. Note that CCAT is on the eastern slope of C. Chajnantor, which provides shielding from the prevailing westerly wind. Measurements have shown lower peak and average wind speeds at the CCAT site than at the ALMA plateau in spite of the $800 \mathrm{~m}$ altitude difference.

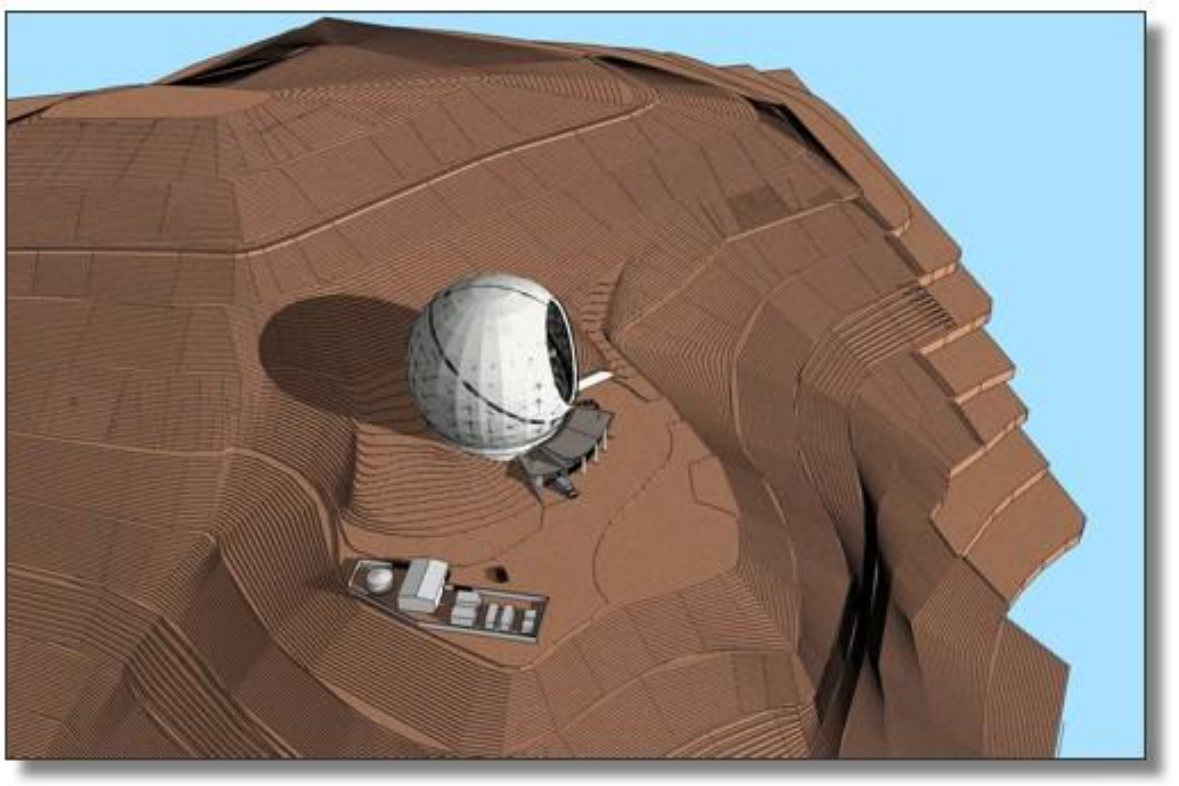

Fig. 5. Topography of CCAT site.
Transparency and meteorological monitoring have been performed continuously on the site over the past 2 years, and results are presented in Simon Radford's paper "Submillimeter observing conditions on Cerro Chajnantor" ${ }^{\text {"[9] }}$ A series of Chajnantor Working Group meetings have been conducted and the issues of logistics in operation at the Atacama Science Preserve are being worked out as the several telescopes now in operation there, ALMA development, and the management efforts of Comisión Nacional de Investigación Cientifica y Tecnológica (CONICYT), who have the concession from the Chilean 
Government to manage the Preserve are reducing the requirements of operating at such high altitudes to practice.

Evolution of the facility design has continued. The major changes since the Study are:

- Lowering of the telescope height to minimize the amount of concrete required.

- Placing the generator plant at lower altitude (the base of C. Chajnanator) to minimized derating, simplify access for maintenance and fuel delivery, and to prepare for access to grid power should that become a possibility in the future.

- Investigation of modular building concepts that might reduce the amount of construction trade work required at the extreme altitude.

- Revisiting the space allocation to ensure that the minimum required facility is constructed at the observatory location.

A survey of approaches to alternative energy was performed at the request of CONICYT. Meteorological data was supplied to wind power development companies and photovoltaic system providers. Strawman systems were devised using the most promising approaches. Unfortunately, data indicated that the break-even point for the cost of development occurred in close proximity to the lifespan of critical system components and that storage technology was not yet adequate for either solar or wind alone to ensure total coverage for the required power. Consequently, the current plan remains to employ two $\sim 750 \mathrm{kVA}$ diesel generators.

\section{TELESCOPE DOME}

Identified as a high-risk area, significant effort has been focused on examining the requirement for an enclosure dome and on the engineering approach to the selected Calotte approach. A study was conducted to evaluate the requirement for the use of an enclosure dome. This study considered data on wind shake, computational fluid dynamics simulations, and wind pressure measurements provided by other astronomical telescope projects. Taking into account the precision pointing requirements of CCAT it was determined that wind driven jitter for an exposed telescope would likely to prevent meeting performance goals. It was also felt that thermal loading on an exposed telescope would make it more difficult to attain the $\sim 10 \mu$ RMS half-wavefront figure error required. Other factors in favor of employing a dome included an extended weather operating envelope, improved survival, reduced cost versus hardening the whole system to survive direct exposure, the need to environmentally house all astronomical instruments, personnel safety, and the additional enclosed space for work and storage.

Additional design work on the dome system was performed by MERO Structures of Wurzburg, Germany.

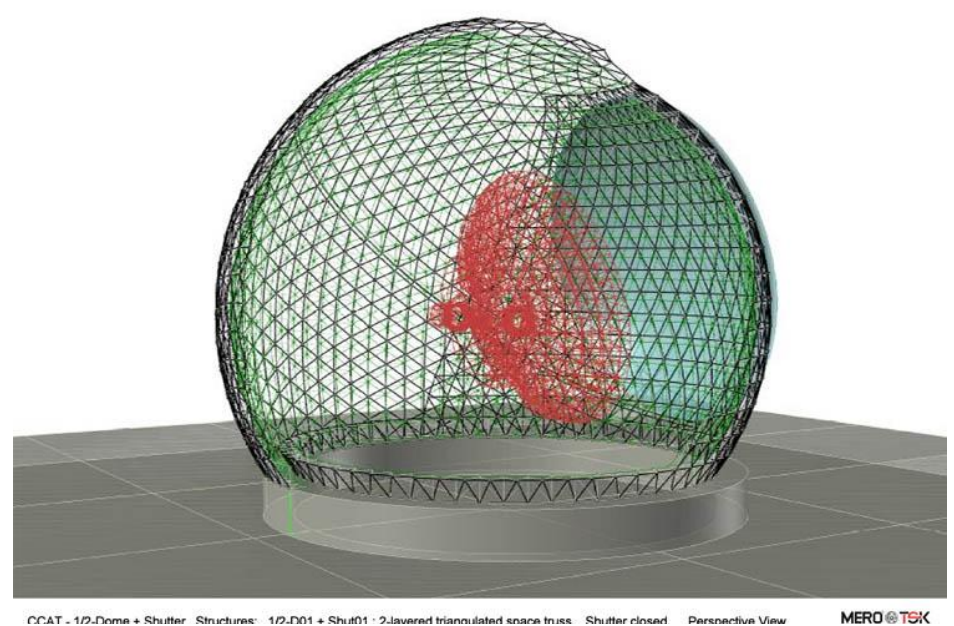

Fig. 6. Geodesic type bolted. Dome structure Concept by MERO structures, Germany.
Where the initial concept provided for the Study by Empire Dynamic Structures (Vancouver, BC) employed a frame of welded struts, the MERO approach uses repetitive geodesic type structure which can be rapidly manufactured using their proprietary robotic manufacturing capability. This dome would pack much smaller for transport to the site, would required little or no field welding, and would achieve dimensional tolerances by way of accuracy of the components rather than field alignment/adjustment.

Note that the new f/ratio for CCAT enabled reduction of the dome size from 50 to 42 meters at the equator. Mechanisms for the dome are still felt to be a critical risk area and further study, design, and analysis will follow in the next phase of development. 


\section{PRIMARY MIRROR}

Significant effort has been put into further refinement of the approach to the 25 meter primary mirror system for CCAT.

\subsection{Segment Position Sensing and Control}

Personnel at JPL and Caltech have developed models for segment tip/tilt/piston control. These models have been used to optimize the segmentation pattern and to investigate the effect of placement and type of edge position sensors. This work has revealed that low order modes are not well controlled by edge sensors alone.

This is particularly significant when front to back thermal gradients occur on the segments, as can occur when the front surface has a view of the cold sky and the rear surfaces face the warm facility. If the gradient varies significantly from segment to segment, the result can be poorly controlled segment positions resulting in unacceptable mirror figure. CCAT's specification is $\sim 10 \mu$ RMS half wavefront error for the three main mirror system. This result has prompted the Project to seek an independent supplementary sensor for mirror tilt sensing.

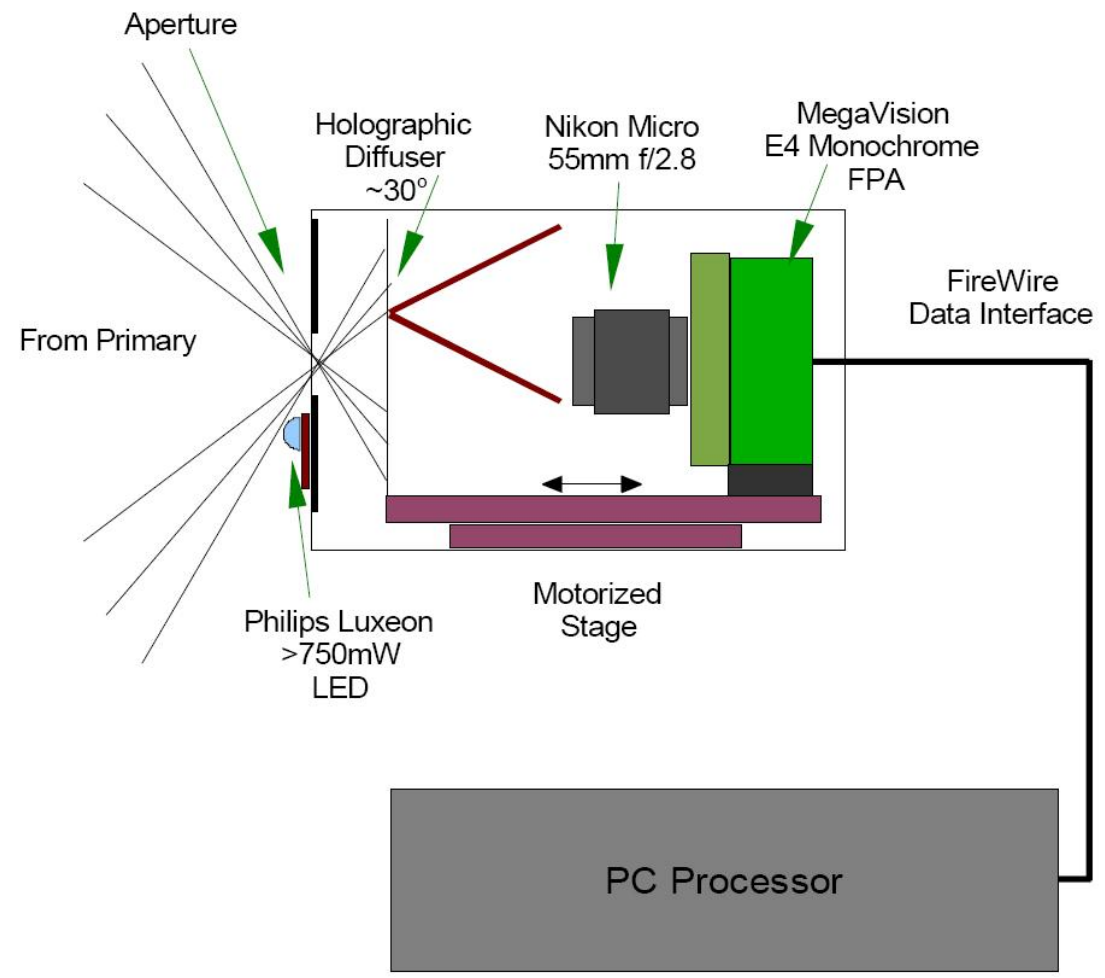

Fig. 7. Concept for optical Segment Tilt Sensor by Adaptive Optics Associates, Cambridge, MA.
A contract to Adaptive Optics Associates in Cambridge, MA resulted in an initial design for a pseudo Shack Hartmann sensor to be located behind the secondary mirror with access via an aperture in the secondary corresponding to the obscuration of the primary by the secondary. A laser diode forms a point source that is reflected by small $(\sim 2.0$ $\mathrm{cm})$ spherical mirrors, one each attached to every segment. These spheres have a radius of curvature equal to the primary to secondary mirror distance. The returning beams converge on a holographic diffuser and the resulting spot pattern is imaged by a high-resolution camera.

The imaging system is translated in focus and images saved at two different conjugates. The relative positions can be reverse ray traced to determine the tip/tilt angles of the segments. There are additional bugs to be worked out, but the possibility of an independent optical mirror tilt sensor remains an area of investigation for CCAT.

\subsection{Segment Fabrication}

Thermal analysis has indicated that the optimal panels for the CCAT segmented primary mirror will employ low CTE materials. For this reason, efforts currently have concentrated on panels formed with CFRP skins and Aluminum honeycomb core. This type of panel has been used successfully with millimeter wave telescope systems. For CCAT, the panels must reach an accuracy of $\sim 5 \mu$ RMS independent of any support structure correction. CCAT has been working to identify vendors with the necessary composites fabrication skills to manufacture such panels. 


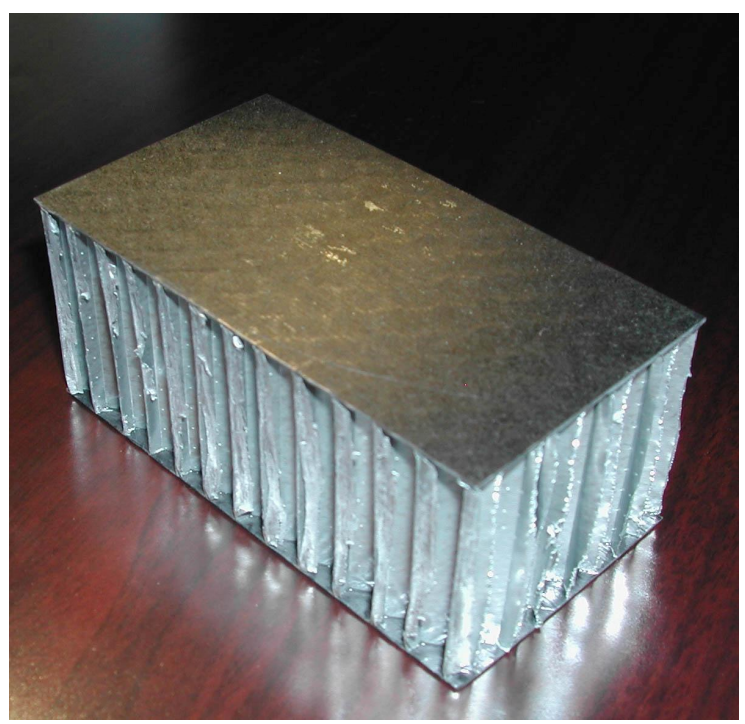

Plans for fabrication anticipate the use of Invar molds fabricated and polished to a precision of about $1 \mu$ RMS. In this approach, panels are mounted kinematically via bipod flexures and are adjustable in rigid body motion only via electromechanical actuators. An alternate approach has recently been identified as an option. In this case, the panels will be supported on multiple points by differential thread adjustment screws to a dimensionally stable support structure. This structure, though stiff and stable, has reasonably straightforward dimension accuracy requirements. It is anticipated that 4-6 panels would be mounted to each of these subassemblies, and the panel shapes optimized by adjustment using a precision Coordinate Measuring Machine (CMM.)

Fig. 8. Sample of Segment Composite Approach by Composite Mirror Applications.

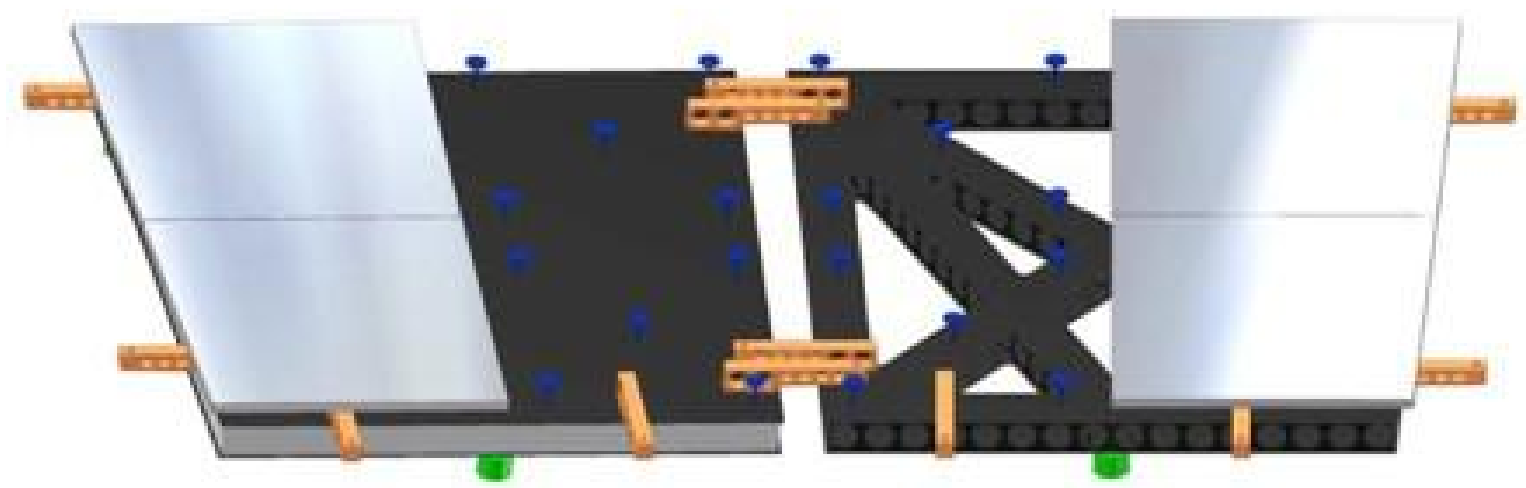

Fig. 9. Concept for "Rafted" type panels for CCAT [10].

\section{PROGRAMMATIC STATUS}

Five partners have signed an Interim Consortium Agreement toward development of CCAT. Work is underway in development of the full partnership agreement, which will govern the development and operation of CCAT. Cornell's Office of the Vice Provost for Research is developing a proposal to host the Project at Cornell and counsel has been retailed by the Project to prepare filing as a not for profit entity. Plans are being developed for an Engineering Design Phase to be performed over the next two years. The objective of this phase is to perform key trades and system engineering, identify and retire critical risks, and prepare for the initial major contracts toward development.

\section{REFERENCES}

[1] Sebring, T., et al., Cornell Caltech Atacama Telescope (CCAT): a $25 \mathrm{~m}$ aperture telescope above $5000 \mathrm{~m}$ altitude, 2006 SPIE Conference, San Diego, CA. 
[2] Radford, S., et al., The Cornell Caltech Atacama Telescope, 18th International Symposium on Space Terahertz Technology, Pasadena, CA (2007).

[3] Laurent, G.T., et al., "The Bolocam Lockman Hole Millimeter-Wave Galaxy Survey: Galaxy Candidates and Number Counts", ApJ, 623, 742 (2005).

[4] Stacey, G.J., et al., "ZEUS: the redshift (z) and early universe spectrometer”, SPIE, 5498, 232 (2004).

[5] Earle, L., et al., "Z-Spec: a broadband direct-detection millimeter-wave spectrometer - instrument statues and first results", SPIE, 6275, 32 (2006).

[6] Irwin, K.D., Hilton, G.C., "Transition-Edge Sensors”, chapter in Cryogenic Particle Detection, C. Enss (Ed.), Topics Appl. Phys., 99, 63-149 (2005).

[7] Day, P.K., LeDuc, H.G., Mazin, B.A., Vayonakis, A., Zmuidzinas, J., “A broadband superconducting detector suitable for use in large arrays", Nature, 425, 817 (2003).

[8] Harrington, J.A, George, R., Pedersen, P., "Hollow polycarbonate waveguides with inner CU coatings for delivery of terahertz radiation”, Optics Express, 12, 5263 (2004).

[9] Radford, S.J.E., Giovanelli, R., Gull, G., Henderson, C.P., "Submillimeter observing conditions on Cerro Chajnantor", SPIE Ground-based and Airborne Telescopes Conference (2008).

[10] Woody, D., MacDonald, D., Bradford, M., Chamberlin, R., Dragovan, M., Goldsmith, P., Radford, S. and Zmuidzinas, J., "Panel Options for Large Precision Radio Telescopes", SPIE Ground-based and Airborne Telescopes Conference (2008). 